CYBERNETICS AND INFORMATION TECHNOLOGIES • Volume 16, No 5

Special Issue on Application of Advanced Computing and Simulation in Information Systems

\title{
PID Controller Design Based on Radial Basis Function Neural Networks for the Steam Generator Level Control
}

\author{
Kong Xiangsong, Chen Xurui, Guan Jiansheng \\ High-Voltage Key Laboratory of Fujian Province, Xiamen University of Technology, Xiamen, China \\ Email: kongxiangsong@gmail.com
}

\begin{abstract}
Steam generator level control system is a vital control system for the Pressurized Water Reactor (PWR). However, the steam generator level process is a highly nonlinear and non-minimum phase system, the conventional ProportionalIntegral-Derivative (PID) control scheme with fixed parameters was difficult to obtain satisfactory control performance. The Radial Basis Function (RBF) Neural Networks based PID control strategy (RBFNN-PID) is proposed for the steam generator level control. This method can identify the mathematical model of the steam generator via the RBF neural networks, and then the PID parameters can be optimized automatically to accommodate the characteristic variation of the process. The optimal number of the hidden layer neurons is also discussed in this paper. The simulation results shows that the PID controller designed based on the RBF neural networks has good control performance on the steam generator level control.
\end{abstract}

Keywords: PID controller, radial basis function, neural networks, steam generator, water level control.

\section{Introduction}

Steam Generator (SG) is the principle interface for the exchange of heat between the primary and secondary side of a Pressurized Water Reactor. Maintain the steam generator level within allowable limits is critical to the safety and economical operation of a nuclear power plant [1]. The level control of the steam generator is vital to achieving the abovementioned goal. However, to improve the control performance of the steam generator level control is a challenging task for the below factors: firstly, the dynamics of the steam generator are highly nonlinear; secondly, the steam generator has non-minimum-phase characteristics, which is called "swell and shrink" phenomena $[2,3]$.

To address the steam generator level control problems, various control schemes have been designed and analyzed. Some promising control schemes have been proposed and established. For example, Irving, Miossec and Tassart 
[4] have proposed a model reference adaptive PID level controller; Feliachi and B elbelidia [5] have proposed a suboptimal controller based on linear output feedback control. With the development of modern computer technology and control theories, various advanced control schemes have been developed to improve the control performance of the steam generator further. Typical works reported in the literature including robust control [6], model predictive control [1, 7], dynamic sliding mode control [8] and intelligent control [9] et al.

In spite of many advanced control methods proposed for the steam generator level control, the controllers widely used in the steam generator level control are still PID controllers. The PID controller is the most commonly used controller in the industrial control field. It has the advantages that it is simple, stable, robust and easy for implementation [10]. The advanced control methods are always complicated and difficult to implement on the real industries. So, there is still a need for higher performance controller which could retain the advantages of the PID controllers as more as possible.

The conventional PID controllers with fixed control parameters have their disadvantages as the parameters are difficult to optimize and they are not suitable for time-varying and nonlinear process. To address the abovementioned problem and design a satisfactory controller, one possible way is to adjust the conventional PID controller structure, and make the parameters of the PID controller to be automatic tuning. The Radial Basis Function (RBF) neural network, which is able to approach a nonlinear function arbitrarily, can be used to identify online steam generator model with high accuracy. In this paper, the RBF neural networks based PID controller has been proposed.

The rest of this paper is organized as follows: Section 2 focuses on the steam generator model. Section 3 introduces the steam generator level control system structure and presents the detail descriptions of the RBF neural networks based PID control scheme. Section 4 illustrates the simulation results of the RBFNN based PID and compares the results with the conventional PID controller, the optimal hidden layer neutrons selection is also analyzed. At last, Section 5 concludes the paper.

\section{Steam generator model description}

To design and verify the control scheme that we proposed, an effective model for the steam generator level process is necessary. The steam generator model, which is proposed by Irving, Miossec and Tassart [4], is used in this paper. This model can capture the essential dynamics of a steam generator, and is simple for control system design. The transfer function of the model is as follows:

$$
Y(s)=\left(\frac{G_{1}}{s}-\frac{G_{2}}{1+\tau_{2} s}\right)\left[Q_{e}(s)-Q_{v}(s)\right]+\frac{G_{3}(s)}{\tau_{1}^{-2}+4 \pi T^{-2}+2 \tau_{1}^{-1} s+s^{2}} Q_{e}(s),
$$

where $Y(s)$ represents the narrow range water level of the steam generator, $Q_{e}(s)$ is the feedwater flow rate, and $Q_{v}(s)$ is the steam flow rate; $T$ is the period of the mechanical oscillation, $\tau_{1}$ and $\tau_{2}$ are the damping time constant; $G_{1}$ is the 
magnitude of the mass capacity effects; $G_{2}$ is the magnitude of the swell and shrink phenomena; $G_{3}$ is the magnitude of the mechanical oscillation.

For this model, the parameters at different power levels had been identified from experimental data by Irving, Miossec and Tassart [4], and they are given in Table 1. It can be seen that the steam generator level process is complicated and nonlinear.

Table 1. Parameter of the steam generator under typical different power levels

\begin{tabular}{|l|c|c|c|c|c|}
\hline \multirow{2}{*}{ Parameters } & \multicolumn{5}{c|}{ Power level $\left(P / P_{n}\right), \%$} \\
\cline { 2 - 6 } & 5 & 15 & 30 & 50 & 100 \\
\hline$G_{1}(\mathrm{~mm} . \mathrm{s}) / \mathrm{kg}$ & 0.058 & 0.058 & 0.058 & 0.058 & 0.058 \\
\hline$G_{2}(\mathrm{~mm} . \mathrm{s}) / \mathrm{kg}$ & 9.63 & 4.46 & 1.83 & 1.05 & 0.47 \\
\hline$G_{3}(\mathrm{~mm} . \mathrm{s}) / \mathrm{kg}$ & 0.181 & 0.226 & 0.310 & 0.215 & 0.105 \\
\hline$\tau_{1}, \mathrm{~s}$ & 41.9 & 26.3 & 43.4 & 34.8 & 28.6 \\
\hline$\tau_{2}, \mathrm{~s}$ & 48.4 & 21.5 & 4.5 & 3.6 & 3.4 \\
\hline$T, \mathrm{~s}$ & 119.6 & 60.5 & 17.7 & 14.2 & 11.7 \\
\hline$Q_{v}, \mathrm{~kg} / \mathrm{s}$ & 57.4 & 180.8 & 381.7 & 660 & 1435 \\
\hline
\end{tabular}

In order to modeling the steam generator level process with MATLAB $S$-Function, the transfer function model in (1) has been converted to the equivalent state space form:

$$
\begin{gathered}
\dot{x}(t)=A(p) x(t)+B(p)(u(t)+d(t)), \\
y(t)=C x(t)+D u(t),
\end{gathered}
$$

where $p$ is the power level, $u(t)$ denotes the $Q_{e}(s), d(t)$ denotes the $Q_{v}(s)$.

The coefficients matrix of (2) is as follows:

$$
\begin{aligned}
& A(p)=\left[\begin{array}{cccc}
0 & 0 & 0 & 0 \\
0 & \frac{-1}{\tau_{2}} & 0 & 0 \\
0 & 0 & -2\left(\frac{1}{\tau_{1}}\right) & 1 \\
0 & 0 & -\left(\frac{1}{\tau_{1}}\right)^{2}-4 \pi^{2}\left(\frac{1}{T}\right)^{2} & 0
\end{array}\right], \\
& B(p)=\left[\begin{array}{cc}
G_{1} & -G_{1} \\
-G_{2} / \tau_{2} & G_{2} / \tau_{2} \\
G_{3} & 0 \\
0 & 0
\end{array}\right], \\
& C(p)=\left[\begin{array}{llll}
1 & 1 & 1 & 0
\end{array}\right] \text {, } \\
& D(p)=\left[\begin{array}{ll}
0 & 0
\end{array}\right] .
\end{aligned}
$$

The water level change in the steam generator is governed by the balance between the flow rates of the incoming feedwater and the exiting steam. Fig. 1 
shows the steam generator level response at two different cases. The response characteristics shows the "swell and shrink" phenomena of the steam generator.
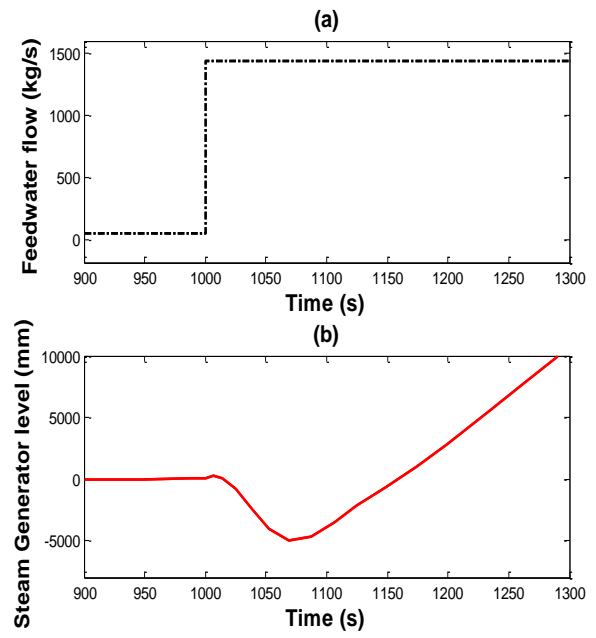

(1)
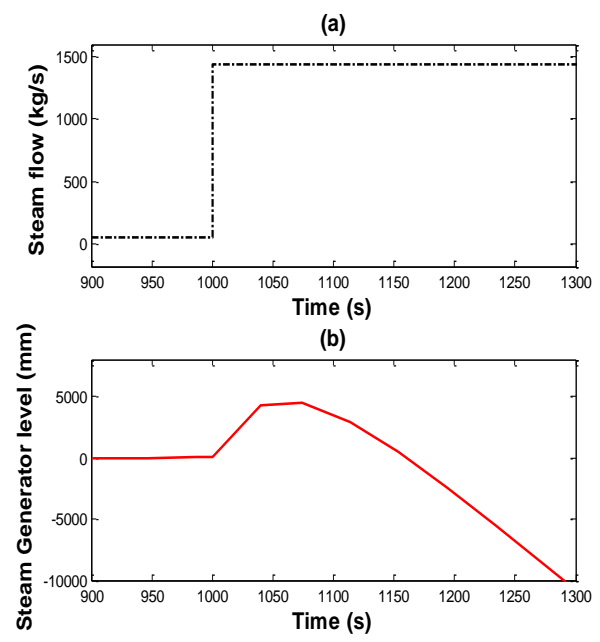

(2)

Fig. 1. Steam generator water level response: The step in feedwater flow (1); the step in steam flow (2)

The main goal of the steam generator level control is to maintain the steam generator water level at a desired value. The complex dynamics of the steam generator significantly complicate the design of the level controller.

\section{PID controller, based on RBF neural networks}

The typical structure of the steam generator level control system is shown in Fig. 2. The controller that is widely used in the nuclear power plant is the PID controller. However, the conventional PID controller has fixed control parameters and is difficult to obtain satisfactory control performance.

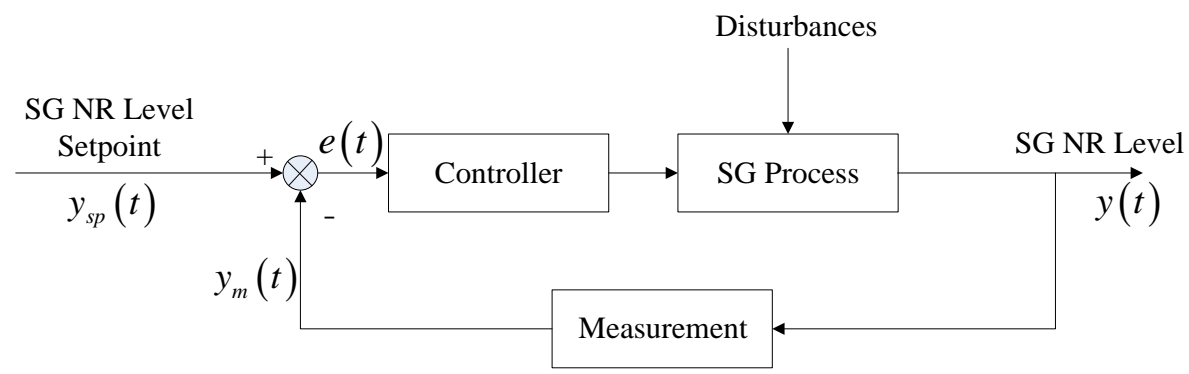

Fig. 2. The structure of the steam generator level control system

An improved PID controller based on the RBF neural networks is proposed. With this method, the control parameters are tuned adaptively according to the 
dynamics of the steam generator. The scheme of the controller is shown in Fig. 3.

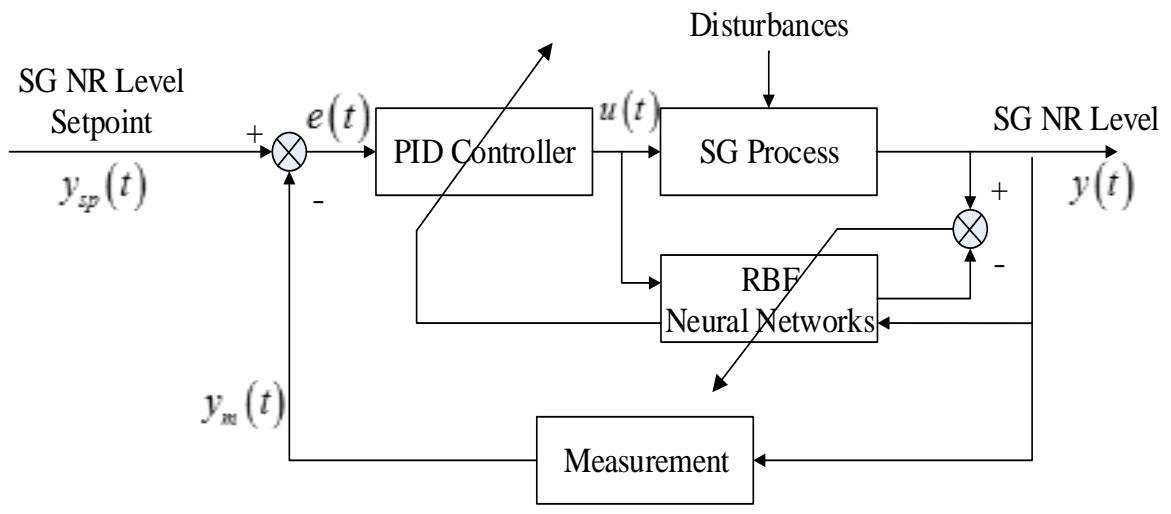

Fig. 3. The structure of the PID control system based on the RBF neural networks

\subsection{RBF neural networks}

RBF Neural Networks (RBFNN) is presented by J. Moody and C. Darken (see $[12,13])$. It is a three-layer feed-forward neural networks with single hidden layer. The mapping from input to output is nonlinear, but from hidden layer to output layer is linear. It has been proved that the RBF network has the ability of approximating any continuous function with any arbitrary accuracy. The learning rate is quickened greatly and the problem of local minimum is avoided. The RBF neural networks configuration for the steam generator control system is shown in Fig. 4.

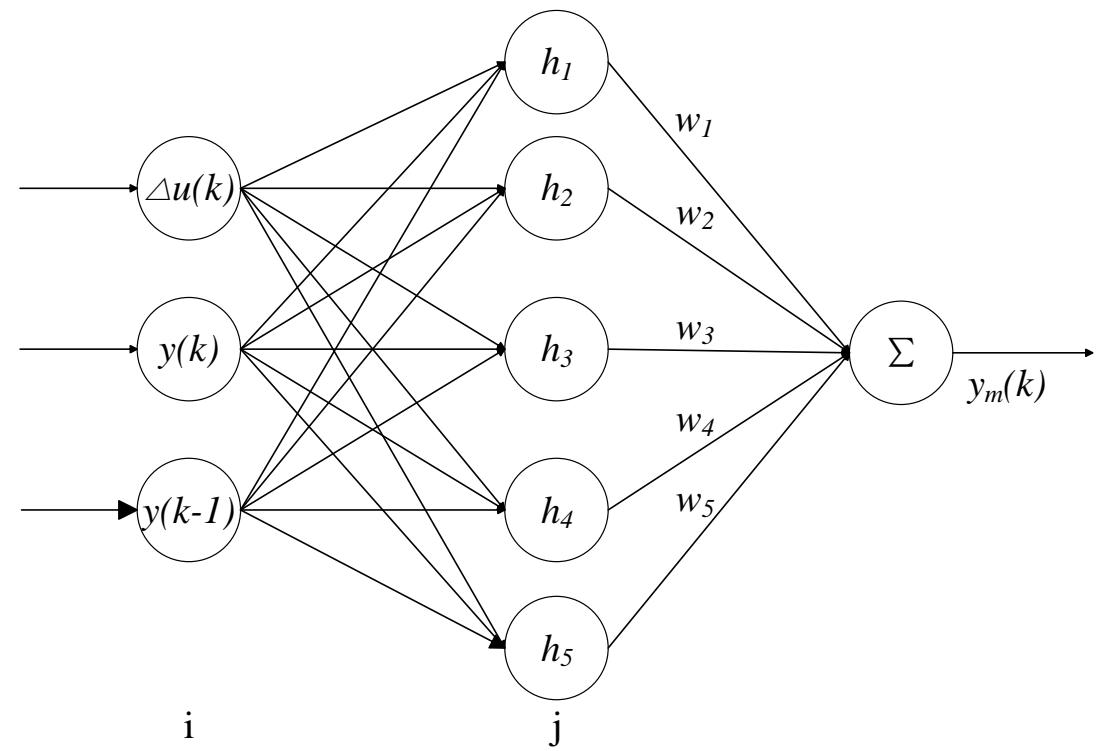

Fig. 4. The structure of the BF neural networks 
Suppose the input vector of the RBF neural networks is $x=\left[x_{1}, x_{2}, \cdots, x_{n}\right]^{\mathrm{T}}$, the neurons at the hidden layer is activated by a radial basis function. Suppose the radial vector is $h=\left[h_{1}, h_{2}, \cdots, h_{m}\right]^{\mathrm{T}}$, where $h_{j}$ is Gaussian function with the following mathematical relation:

$$
h_{j}=\exp \left[-\frac{\left\|x-C_{j}\right\|^{2}}{2 b_{j}^{2}}\right], j=1,2, \cdots, m .
$$

The center vector of the network at node $j$ is $C_{j}=\left[c_{j 1}, c_{j 2}, \cdots, c_{j i}, \cdots, c_{j n}\right]^{\mathrm{T}}, i=1,2, \cdots, n$. Suppose the radial width vector is $B=\left[b_{1}, b_{2}, \cdots, b_{m}\right]^{\mathrm{T}}$, where $b_{j}$ is the radial parameter of node $\mathrm{j}$, and $b_{j}>0$. The weight vector of the network is $W$ and $W=\left[w_{1}, w_{2}, \cdots, w_{m}\right]^{\mathrm{T}}$.

The output of the RBF networks can be calculated as following:

$$
y_{m}(k)=W^{\mathrm{T}} h=w_{1} h_{1}+w_{2} h_{2}+\cdots+w_{m} h_{m} .
$$

The performance index function is

$$
E(k)=\frac{1}{2}\left(y(k)-y_{m}(k)\right)^{2},
$$

where $y(k)$ is the output of the system at $k$.

According to the gradient descent algorithm, the iterative algorithm of output width, center joint and joint base width is as follows:

$$
\begin{gathered}
\Delta w_{j}(k)=\eta\left(y(k)-y_{m}(k)\right) h_{j}, \\
w_{j}(k)=w_{j}(k-1)+\Delta w_{j}(k)+\alpha\left(w_{j}(k-1)-w_{j}(k-2)\right), \\
\Delta b_{j}(k)=\eta\left(y(k)-y_{m}(k)\right) w_{j} h_{j} \frac{\left\|X-C_{j}\right\|^{2}}{b_{j}^{3}}, \\
b_{j}(k)=b_{j}(k-1)+\Delta b_{j}+\alpha\left(b_{j}(k-1)-b_{j}(k-2)\right), \\
\Delta c_{j i}(k)=\eta\left(y(k)-y_{m}(k)\right) w_{j} \frac{x_{j}-c_{j i}}{b_{j}^{2}}, \\
c_{j i}(k)=c_{j i}(k-1)+\Delta c_{j i}+\alpha\left(c_{j i}(k-1)-c_{j i}(k-2)\right),
\end{gathered}
$$

where $\alpha$ is the learning rate, $\eta$ is the momentum factor. In this project, $\alpha=0.05, \eta=0.25$.

The Jacobian algorithm is

$$
\frac{\partial y(k)}{\partial \Delta u(k)} \approx \frac{\partial y_{m}(k)}{\partial \Delta u(k)}=\sum_{j=1}^{m} w_{j} h_{j} \frac{c_{j i}-x_{1}}{b_{j}^{2}}
$$

where $x_{1}=\Delta u(k)$. 


\subsection{RBFNN based PID controller}

The incremental PID controller is adopted. The control error is

$$
e(k)=y_{d}(k)-y(k) \text {. }
$$

The three inputs of the controller are as follows:

$$
\begin{gathered}
x c(1)=e(k)-e(k-1), \\
x c(2)=e(k), \\
x c(3)=e(k)-2 e(k-1)+e(k-2) .
\end{gathered}
$$

Then the incremental PID control algorithm is

$$
\Delta u(k)=k_{p}[e(k)-e(k-1)]+k_{i} e(k)+k_{d}[e(k)-2 e(k-1)+e(k-2)] \text {. }
$$

Neural network-tuning of indicators is as follows:

$$
E(k)=\frac{1}{2} e(k)^{2} .
$$

The control parameters of the PID controller are adjusted based on the gradient descend method are as follows:

$$
\begin{gathered}
\Delta k_{p}=-\eta \frac{\partial E}{\partial k_{p}}=-\eta \frac{\partial E}{\partial y} \frac{\partial y}{\partial \Delta u} \frac{\partial \Delta u}{\partial k_{p}}=\eta e(k) \frac{\partial y}{\partial \Delta u} x c(1), \\
\Delta k_{i}=-\eta \frac{\partial E}{\partial k_{i}}=-\eta \frac{\partial E}{\partial y} \frac{\partial y}{\partial \Delta u} \frac{\partial \Delta u}{\partial k_{i}}=\eta e(k) \frac{\partial y}{\partial \Delta u} x c(2), \\
\Delta k_{d}=-\eta \frac{\partial E}{\partial k_{d}}=-\eta \frac{\partial E}{\partial y} \frac{\partial y}{\partial \Delta u} \frac{\partial \Delta u}{\partial k_{d}}=\eta e(k) \frac{\partial y}{\partial \Delta u} x c(3),
\end{gathered}
$$

where $\frac{\partial y}{\partial \Delta u}$ is the Jacobian information of controlled steam generator level process, and it can be identified by the RBF neural networks.

The structure of PID controller based on the RBF neural networks is shown in Fig. 3 .

\section{Simulation results and discussions}

All the simulations were conducted on the steam generator model that was built based on the MATLAB and Simulink according to (2)-(6). To test the effectiveness of the propose RBFNN based PID controller, the control performance of the RBFNN based PID was compared with the conventional PID in a qualitative and a quantitative way, respectively. Considering the number of the hidden layer neutrons has significant effect on the performance of the RBFNN based PID controller, the optimal hidden layer neutrons number was analyzed. 
Without loss of generality, the level setpoint of the steam generator is set to $0 \mathrm{~mm}$. The disturbance transient is $10 \%$ step increase of steam flow, which occurs at the $1000 \mathrm{~s}$ of a simulation.

\subsection{Qualitative analysis}

Two cases were tested with different power levels. The 1st test was under 5\% power level. For the 1st test, two simulations with different control schemes were implemented. The 2nd test was under $15 \%$ power level with two different simulations too. The water level response from the $1000 \mathrm{~s}$, when the disturbance occurs, is shown in Fig. 5.

The simulation results show that the propose RBF neural networks based PID is better than the conventional PID. The settling time of transient under the same power level is significantly decreased. The different water level responses indicated the nonlinearity of the steam generator. The good performance under different power levels ensured that the RBFNN based PID was adapted for the process nonlinear.

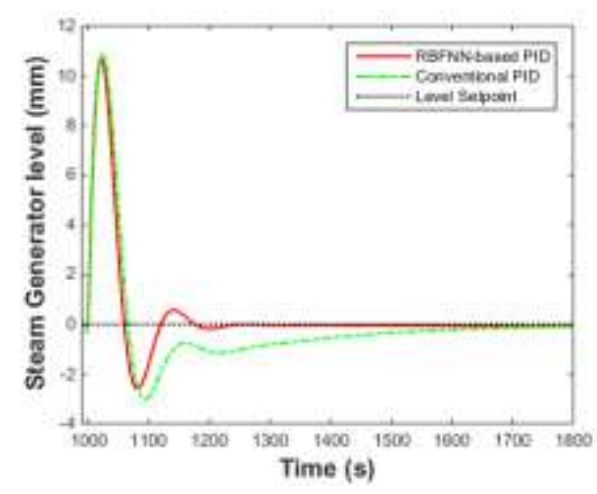

(1)

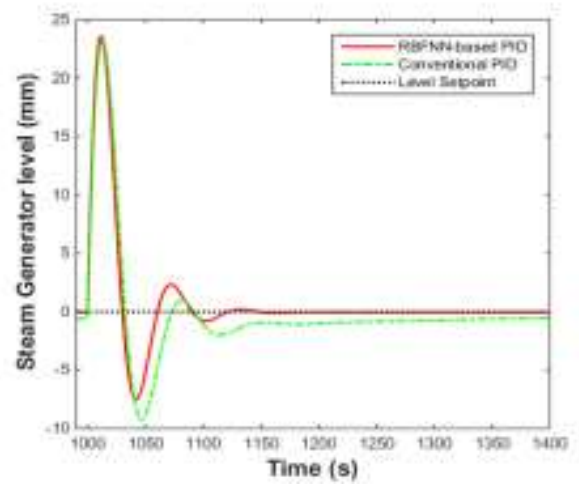

(2)

Fig. 5. Water level response with different control schemes: 5\% power level (1); 15\% power level (2)

\subsection{Quantitative analysis}

To further evaluate the performance of the RBF neural networks-based PID controller, quantitative analysis is needed. First of all, an index is needed for the evaluation.

For a control system, regulation performance is often expressed in terms of the control error obtained for certain disturbances. Typical control evaluation index can be expressed as below:

$$
I=\int_{0}^{\infty} t^{n}|e(t)|^{m} d t
$$

where the error is defined as $e=y_{s p}-y(t), y_{s p}$ is the target and $y(t)$ is the actual response. 
In Table 2, the ITAE calculated results were summarized. The two comparative results at the same power level were obtained under the same conditions. The corresponding figure is Fig. 6. From the figure, it can be seen that the ITAE for the RBFNN-PID was much lower than the conventional PID at the same power level. The lower the ITAE index, the better the control performance.

Table 2. ITAE comparison between conventional PID and the RBFNN-based PID
\begin{tabular}{|c|c|c|}
\hline Power Level & ITAE of Conventional PID & ITAE of RBFNN-PID \\
\hline $100 \%$ & $1.91 \times 10^{6}$ & $2.31 \times 10^{5}$ \\
\hline $15 \%$ & $4.01 \times 10^{5}$ & $1.62 \times 10^{3}$ \\
\hline $5 \%$ & $5.52 \times 10^{5}$ & $1.14 \times 10^{4}$ \\
\hline
\end{tabular}

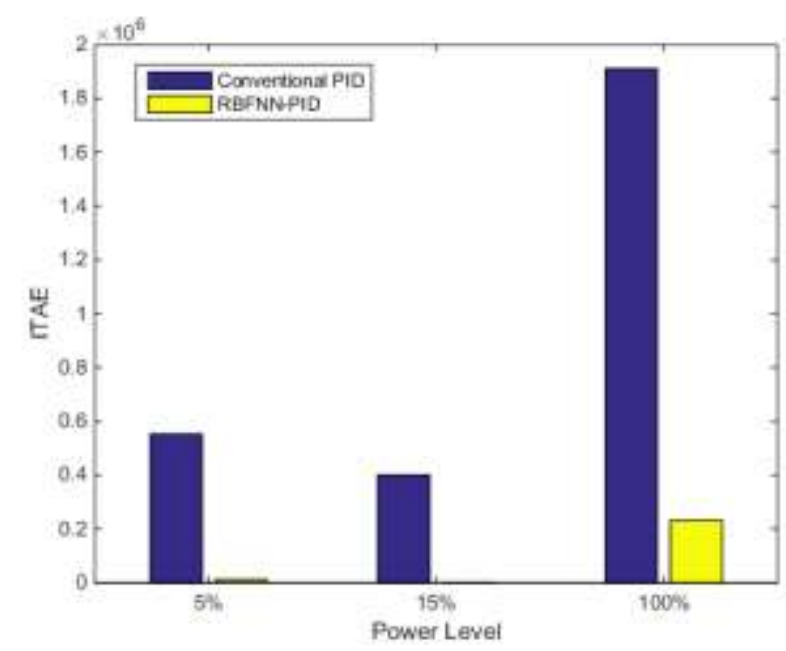

Fig. 6. Control performance comparison between conventional PID and the RBFNN-based PID

\subsection{Optimal hidden layer nodes number analysis}

The number of the hidden layer nodes has significant influence on the performance of the RBF neural networks based PID controller. The performance of the controller has two impacts: The control accuracy and the running time.

Fig. 7 shows the water level transient response under different number of hidden layer nodes. Fig. 8 shows the ITAE trajectory under different hidden layer nodes. It can be seen from the figures that when the number of hidden layer nodes increases, the accuracy improves at the beginning; however, when the number was too large, the control accuracy got worse, the learning process of the controller began to oscillate and the settling time increased.

Fig. 9 shows the running time of the RBFNN-PID at different number of hidden layer nodes. It is obvious that when the number of nodes increases, the running time of the controller increases nearly in proportional to the number of the nodes. 


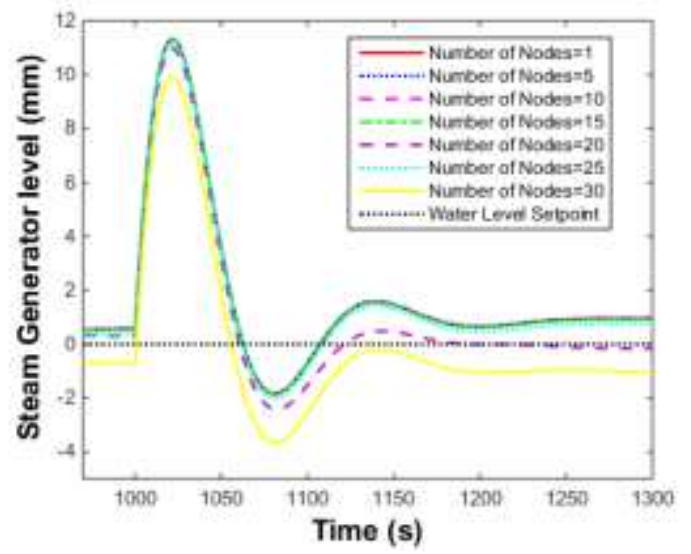

Fig. 7. Water level responses under the RBFNN-PID with different number of nodes

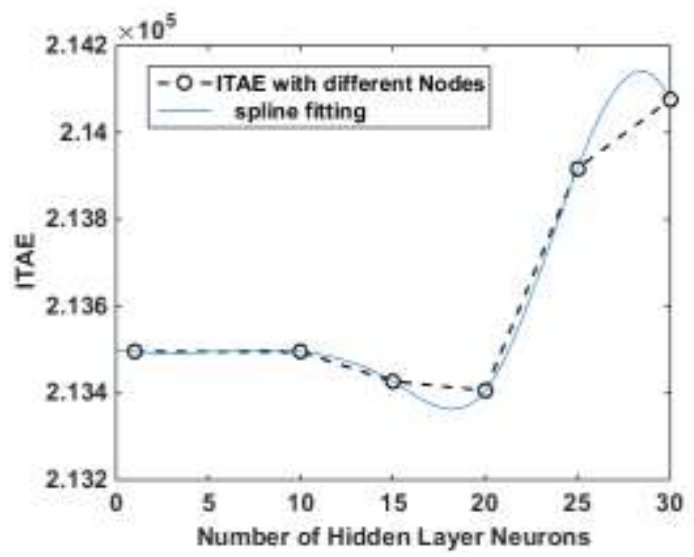

Fig. 8. Control performance indicated by ITAE at different number of nodes

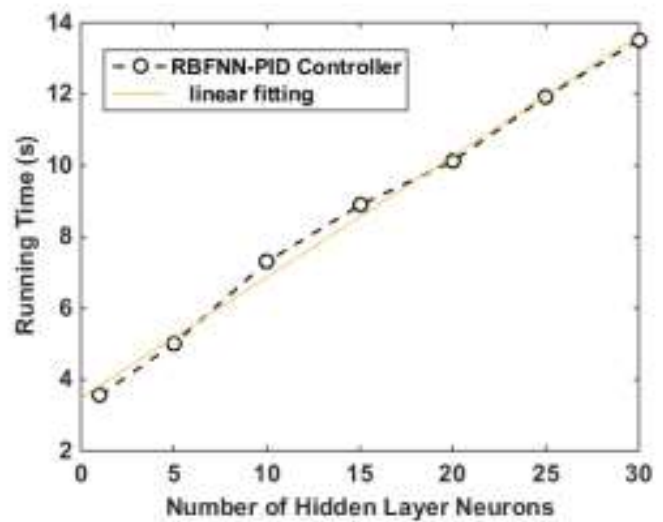

Fig. 9. Running time of the RBFNN-PID at different number of nodes 
According to these results, the optimal number of hidden layer nodes should be neither too large nor too small. Trade-offs should be made between the accuracy and the running time. In this project, the hidden layer nodes was optimized and set to five.

\section{Conclusion}

This paper focuses on the steam generator level control system, which was formulated based on the PID controller. The RBF neural networks based PID control strategy was designed and implemented on the steam generator level control. The RBF neural networks is used to identify the Jacobian information of the controlled level process. And the PID parameters was tuned based on the Jacobian information. The input of the RBF neural networks is selected as three, and the output of RBF neural network acts as traditional PID controller with selfadaptive capability through the RBF neural networks learning and training. Therefore, combined both merits of PID controller and RBF neural network, the RBF neural networks based PID controller has excellent anti-disturbance and adaptively.

The comparisons of the proposed controller with the conventional PID controller shows that the RBF neural networks based PID has better performance. Simulation results indicated the effectiveness of the RBF neural networks based PID controller.

Acknowledgments: We are grateful and wish to acknowledge the financial support of the Scientific Research Fund of Fujian Provincial Education Department (JA15358) and the Xiamen University of Technology High Level Talents Projects (YKJ13034R).

\section{References}

1. M a y u res h, V. K. Level Control in the Steam Generator of a Nuclear Power Plant. - IEEE Transactions on Control Systems Technology, Vol. 8, 2000, No 1, pp. 55-69.

2. A b l a y, G. Robust Estimator-Based Optimal Control Designs for U-Tube Steam Generators. Transactions of the Institute of Measurement and Control, Vol. 37, 2015, No 5, pp. 636-644.

3. F a n g, F., Y. X i o n g. Event-Driven-Based Water Level Control for Nuclear Steam Generators. IEEE Transactions on Industrial Electronics, Vol. 61, 2014, No 10, pp. 5480-5489.

4. Irving, E., C. Mios s e c, J. T a s s art. Toward Efficient Full Automatic Operation of the PWR Steam Generator with Water Level Adaptive Control. - In: Proc. of 2nd Int. Conf. Boiler Dynamics and Control in Nuclear Power Stations, Bournemouth, U.K., October 1979, pp. 309-329.

5. Felia chi, A., L. A. B elbelidia. Suboptimal Level Controller for Steam Generators in Pressurized Water Reactors. - IEEE Trans. Energy Convers. Vol. 3, 1988, pp. 278-284.

6. P a r 1 o s, A. G., O. T. R a i s. Nonlinear Control of U-Tube Steam Generators via $\mathrm{H} \infty$ Control. Control Eng. Pract., Vol. 8, 2000, pp. 921-936.

7. N a, M. G. Auto-Tuned PID Controller Using a Model Predictive Control Method for the Steam Generator Water Level. - IEEE Trans. Nucl. Sci., Vol. 48, 2001, pp. 1664-1671.

8. A n s a rif a r, G. R. Control of the Nuclear Steam Generators Using Adaptive Dynamic Sliding Mode Method Based on the Nonlinear Model. - Annals of Nuclear Energy, Vol. 88, 2016, pp. 280-300. 
9. N a, M. G. Design of Genetic Fuzzy Controller for the Nuclear Steam Generator Water Level Control. - IEEE Trans. Nucl. Sci., Vol. 45, 1998, pp. 2261-2271.

10. Å s t r ö m, K. J., T. H äg g 1 u n d. PID Controllers: Theory, Design and Tuning. 2nd Ed. Research Triangle Park, NC: Instrum. Soc. Amer., 1995.

11. H a y k i n, S. Neural Networks: A Comprehensive Foundation. 3rd Ed. NJ, USA, Prentice-Hall, Inc., 2007.

12. W a n g, J.-J., C.-F. Z h a n g, Y.-Y. J i n g. Self-Adaptive RBF Neural Network PID Control in Exhaust Temperature of Micro Gas Turbine. - In: Proc. of 7th International Conference on Machine Learning and Cybernetics, Vol. 4, July 2008, Kunming, China, pp. 12-15.

13. E 1 a n a y a r, S. V. T., Y. C. S h i n. Radial Basis Unction Neural Network for Approximation and Estimation of Nonlinear Stochastic Dynamic Systems. - IEEE Transaction on Neural Network, Vol. 5, 1994, No 4, pp. 584-603. 